\title{
SIOT FOR COGNITIVE LOGISTICS: LEVERAGING THE SOCIAL GRAPH OF DIGITAL TWINS FOR EFFECTIVE OPERATIONS ON REAL-TIME EVENTS
}

\author{
Miha Cimperman ${ }^{1}$, Angela Dimitriou $^{2}$, Kostas Kalaboukas $^{3}$, Aziz S. Mousas ${ }^{4}$, Salvatore Quattropani ${ }^{5}$ \\ ${ }^{1}$ Institute Jozef Stefan, Ljubljana, Slovenia,${ }^{2}$ Intrasoft International SA, Luxemburg, ${ }^{3}$ Technical University of Crete, \\ Greece, ${ }^{4}$ SingularLogic SA, Greece, ${ }^{5}$ CNIT - RU at the University of Catania, Italy
}

NOTE: Corresponding author: Salvatore Quattropani, Salvatore.quattropani@cnit.it

\begin{abstract}
Over the years, with the migration of organizations towards the concepts of logistics 4.0, a paradigm shift was necessary to guarantee logistics efficiency. The challenge is to dynamically cope in real time with vast number of shipments and destinations, which need to be realigned both with a determined lead time and with a finite of available resources. Although a number of standards have already been adopted for the management of transport and logistics operations, taking advantage, for instance, of Decision Support Systems and Geographic Information Systems, new models are required for achieving effective handling of the dynamic logistics environment that is shaped today. In this paper, an integrated logistics framework addressing the previous challenges is presented, for the first time, as a result of the activities of the H2020 COG-LO project. This novel approach exploits Social Internet of Things (SIoT) and the digital twins technique to realize the concept of the Cognitive Logistics Object (CLO). A CLO is defined as an entity that is augmented with cognitive capabilities, it is autonomous, and bears social-like capabilities, which enable the formulation of ad hoc communities for negotiating optimal solutions in logistics operations.
\end{abstract}

Keywords - Cognitive logistics, collaborative logistics, digital twins, Social Internet of Things

\section{INTRODUCTION}

The development of IoT and cyber-physical systems [1] technologies lay the foundations for the evolution of logistics 4.0, in which the way of organizing supply and production changed drastically. The digitization process pushes logistics operators and all interested parties to turn towards an approach that embraces all the technological innovations that the market offers. The aim is to reach the economic objectives faster and to improve logistics services' quality. The new emerging logistics scenario requires rapid information processing with a high level of security. The spread of big data, the expansion of the logistics chain, the problem of route optimization, the localization of resources, and the maximization of the load factor have become the main focus of research and development in the logistics sector. The challenges listed do not only require the introduction of new logistics concepts, but also need a significant effort to go beyond the common vision of the elements participating in the logistics chain. The COG-LO project [2] addresses these challenges by implementing innovative tools that enable logistics 4.0 [3]. Logistics entities become both cognitive and collaborative. Their level of interoperability increases significantly thanks to the digitization of all the actors participating in the supply chain (cargo, vehicle, warehouse, parking slot, other transport modes, systems, etc.). This is achieved by exploiting an ad hoc dynamic social network based on the Social Internet of Things (SIoT) paradigm [4]. SIoT enables these actors to interact and negotiate potential alternative solutions to emerging logistics requirements, taking into account their current status, needs and identified limitations. The proposed solution implements dynamic optimization strategies streamlining the burden of the decision-making process, enhancing the robustness of artificial intelligence systems, and thus allowing an increasingly pervasive approach of these techniques within the logistics 4.0 world.

The document is organized as follows: In Section 2, the necessary background information is provided. In Section 3, the COG-LO framework is introduced. In Section 4, the system's performance is evaluated. Finally, Section 5 concludes with some final remarks.

\section{DIGITAL LOGISTICS INFRASTRUCTURE}

In recent years, the issues of digital transformation of transport infrastructures have been of particular importance in the context of Industry 4.0. Numerous real-time planning algorithms have been developed by the logistics community over the last thirty years; these include Decision Support Systems (DSS) [5] and Geographic Information Systems (GIS), i.e. a group of procedures that provide input, data storage and retrieval, mapping and spatial analysis [6], and tools to support the organization's decision-making activities. In the past decades, several efforts have been made to integrate GIS with DSS, promoting the concept of collaborative GIS. The GS1 [7] system of standards is currently the definitive framework widely recognized in the field of logistics. It ensures a high degree of interoperability between stakeholders and provides a standard way to identify objects and locations. 
Despite the enormous bene its of the aforementioned systems, there are still many limitations. Most dynamic routing algorithms are unable to analyze and distinguish the nature of external events affecting the supply chain, rendering therefore these systems unable to perform periodic re-optimizations [8]. In consequence, new models are needed that move away from the concepts on which traditional (functional) logistics are based.

\subsection{Digital twin - Cognitive Logistic Object}

The concept of Digital Twins (DT) has been introduced by M. Grieves [9] as a digital representation of a physical entity, for which a specific behaviour can be modelled. After performing appropriate simulations and calculations on this behaviour an action may be triggered on that entity. In the basis of the DTs, various scenarios have been introduced at the manufacturing, logistics sector [10], health and other sectors [11]. As the DT becomes a strong technological trend [12], with companies tending to invest on digital transformation, the transition to a DT modelling approach in logistics can offer new collaborative models and optimization procedures in the domain. The application of the DT paradigm in logistics has been introduced by the concept of a Cognitive Logistics Object [13]. A Cognitive Logistics Object (CLO) is a virtualized object (similar to a DT) or system that participates in the logistics process. It exhibits properties like autonomy, context awareness, responsiveness and learning ability. The CLO represents different actors such as cargo, truck, traffic light, supporting system, etc., with each one having different capabilities. In particular, a CLO is an autonomous object, reactive to changes in the environment and its context. It is able to learn, collaborate, decide on next actions, create social networks and solve local problems. Thanks to the virtualization of objects, communication between heterogeneous systems becomes possible, and each CLO action takes into account various variables such as business priorities, environmental conditions, traffic conditions, load information etc.. Furthermore, each virtualized entity implements the functionalities required for managing the entity's communications. A CLO exhibits social behaviour, which means that the digital counterpart of the logistics object implements a series of services offered by SIoT to establish relationships with other CLOs, dynamically exchange information and thus optimize logistics operations. The virtualization-based approach is quite common in the IoT domain [14] as it promotes interoperability and extends an object's physical and digital characteristics.

\subsection{Social Internet of Things}

The SIoT paradigm brings social network concepts to the IoT context. According to this paradigm, each object is characterized by a social behavior and therefore its digital twin is capable of creating social relations on the basis of common elements and affinity. In the resulting social network, any object looks for desired services by using its relationships, querying its friends and the friends of its friends in a distributed manner. This procedure guarantees an efficient and scalable discovery of CLOs and services following the same principles that characterize the social networks for humans. The following types of relationships are indicative:

- Ownership Object Relationship (OOR): created between objects that belong to the same owner.

- Co-location Object Relationship (CLOR): created between stationary devices located in the same place (also called Co-Geolocation CGLOR).

- Parental Object Relationship (POR): created between objects of the same model, producer and production batch.

- Co-Work Object Relationship (CWOR): created between objects that meet each other at the owner's workplace (e.g., two trucks parked at a depot).

- Social Object Relationship (SOR): created as a consequence of frequent meetings between objects.

- Transactional Object Relationship (TOR): established between devices that interact with each other frequently [15].

- Time Plan Object Relationship(TPOR): created between CLOs that have coincident or overlapping schedules.

The social graph generated by the SIoT in the COG-LO context is an undirected graph of CLOs connected with the aforementioned relationships. It is similar to a social graph generated by friendships between humans with common characteristics. The navigability problem of a social network has been widely addressed by Milgram, and the small world phenomenon [16] has been at the centre of social science research for decades. According to Milgram's hypothesis, even if a social graph is very large and two nodes are very distant from each other, it is possible, starting from one, to reach the other by surfing the net in less than 6 hops, thanks to the existence of short paths between pairs of nodes. The SIoT is responsible for establishing relationships on the basis of local information, therefore creating the necessary conditions for social navigation of the CLOs' graph. To achieve this, advanced storage techniques are adopted to facilitate navigability and ensure that the connections between the nodes of a graph on a logical level are equally accessible on the data plane [17]. To meet this challenge, SIoT exploits the metadata of the social nodes to efficiently index every single data, connection or path in the graph. SIoT can support an MLbased optimizer capable of pruning the social graph in order to generate a smaller subgraph, which represents some elements of Milgram's small world, where nodes have high correlation based on their local information. According to the principles explained above, each logistics object is associated with a CLO, which is the digital 
representation of the physical entity that implements the functionality required for managing the communications and for supporting the common control plane [17]. A CLO carries metadata associated with a given object, such as information on object's nature, status and list of friends. Among the information characterizing the CLO, semantic descriptions facilitate the interactions between digital twins, supporting the detection and management operations despite devices heterogeneity. A CLO, as a software entity, can be implemented in SIoT repositories and hosted in the cloud or on the edge of the network infrastructure. SIoT is responsible for managing the life cycle of each digital twin, store and update their status information in real time and disseminating data from the digital twin to the physical world through a new data communication delivery method and scheme called Sociocast [18]. In particular, Sociocast leverages the SIoT concept to support group communications among nodes in an efficient and effective way on the control plane. With data driven logistics, dynamic optimization of basic logistics processes is at the forefront of the next generation of logistics services. Finding optimal routes for vehicles is a problem that has been studied for many decades from a theoretical and practical point of view. What is typically associated with the Vehicle Routing Problem (VRP) is a generalization of the Travelling Salesperson Problem, where multiple vehicles are available. This class of routing problems is notoriously hard; it not only falls into the class of NP-complete problems, but also it cannot be solved optimally in practice, even for moderate instance sizes. More importantly, processing VRP optimization on large graphs in real-time demands employing additional techniques, such as heuristics and/or graph pruning. Different clustering approaches have been used in pruning the input graph for VRP. For example, Ruhan et al. [19] uses k-means clustering in combination with a rebalancing algorithm to obtain areas with balanced numbers of customers. Bent et al. also study the benefits and limitations of vehicle and customer-based decomposition schemes [20], demonstrating better performance with the latter. In COG-LO, linear optimization was applied as an exact optimization approach for solving VRP. The combination of an enriched social-like behavior and instant-by-instant knowledge of an object's state, allows SIoT to support requests from optimization systems by effectively pruning CLO graphs on the basis of social relationships. Therefore, it proves to be a critical part for real-time optimization.

\subsection{SIoT platform}

Conceptually, the Social Internet of Things (SIoT) platform consists of various clusters of SIoT peers. Each SIoT peer is made up of different functional blocks and exposes its data and services via REST calls (Fig. 1).

The Cognitive Logistic Objects Repository (CLOR) is a data structure that contains all CLO information. Friend Tables (FTs) are the data structure where the CLO
Friends information is hosted. The SIoT hosts only one Friend Table for each CLO. The FT content represents the CLO friends and contains information about the friendships. The Relationship Manager (RM) module implements the logic through which a relationship can be created, deleted or updated. It is responsible for providing rules for implementing the social relationships among IDlabelled CLOs. When a new relationship is created, the RM writes directly in the Friend Table distributed database of the involved CLOs. The Relationship Browser (RB) module deals with navigating the social graph while implementing the search algorithms. In particular, the RB deals with the analysis of the FTs to find a target destination/set of destinations to be reached among the friends of a given entity. The Identity Service (IS) module is responsible for managing the Identifiers lifecycle. IS manages the IDs of the created or removed CLOs. Subscription Service (SS) allows to the peer or cluster RM to subscribe to information about CLO changes (for example position) and provides support for pushing the relevant updates. SS is exploited whenever the friendship tables of the CLOR managed by two different RMs need to be updated. The Virtual Instance (VI) mdule represents the virtual instance of CLO: when a CLO has to interact, make decisions or perform computation, this component is instantiated. It is released when the CLO gets idle. It represents the digital twin of the CLO, extends its capabilities and carries out social behaviours.

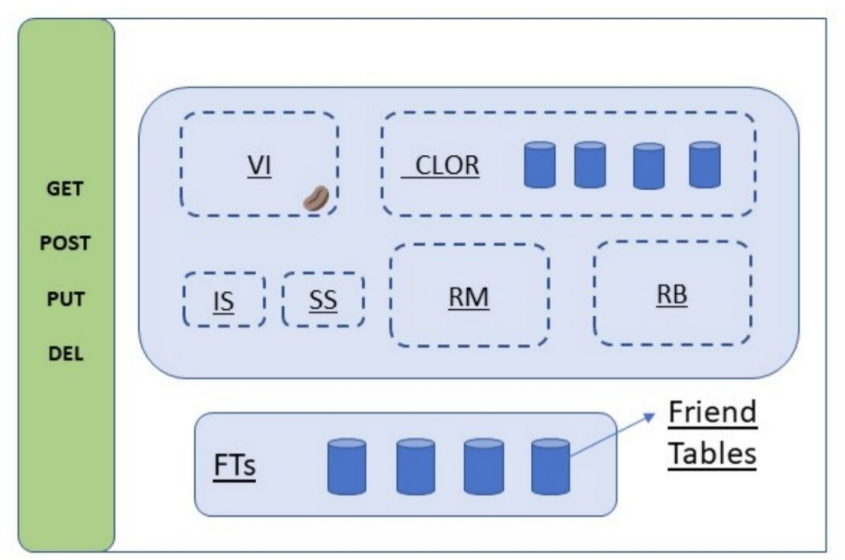

Fig. 1 - SloT peer

\section{COG-LO FRAMEWORK}

The COG-LO framework aims to provide a holistic solution for handling the high operational dynamicity of the logistics environment. It follows a layered architecture and provides interoperable solutions regarding data models, information exchange and security mechanisms. This way it enables transparent coordination and exchange of information between different objects and systems based on heterogeneous access protocols in a secure manner. 


\subsection{Architecture}

The physical entities that collectively carry out the actual logistics processes are denoted as the Infrastructure layer. These refer to a variety of concepts, including cargo (parcel, palette, container, etc.), transportation means (vehicles, trucks, trains, etc.), back-end ICT systems and services, as well as infrastructure components, like hubs, parking places, ports and other. The entities of the infrastructure are complemented by the datasources, including any source of data such as Enterprise Resource Planning, Warehouse Management System, Intelligent Transportation Systems, Traffic Information Systems, along with operational and configuration data that are essential for the operation of COG-LO.

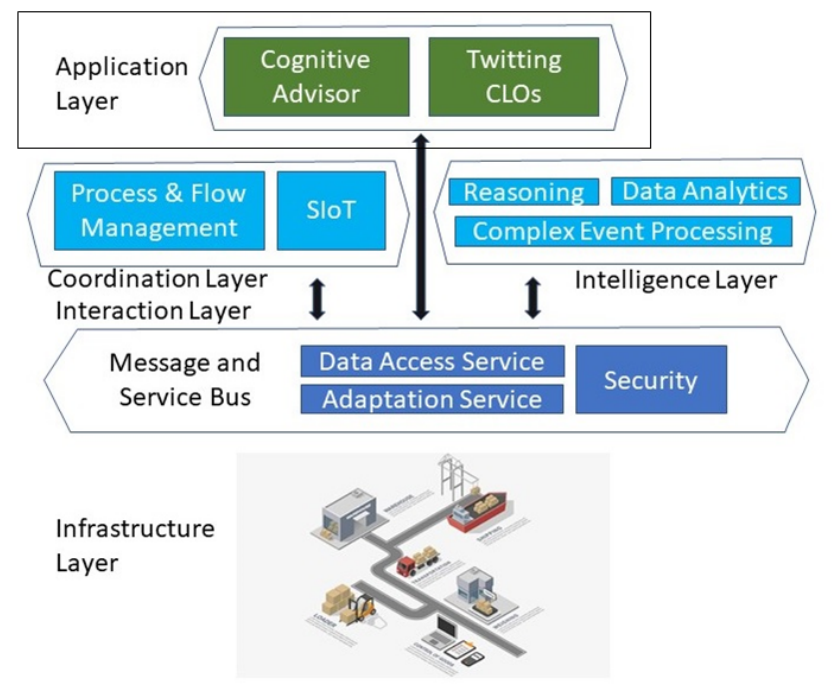

Fig. 2 - COG-LO Architecture

In order to provide for effective interaction, an Interaction Layer has been created. It includes a Message and Service Bus (MSB) which acts as mediation middleware between the components of the COG-LO ecosystem. The MSB comprises a message-oriented system providing both asynchronous and point-to-point message exchange between the system entities, circulation of events, and interaction between the CLOs. Furthermore, the MSB provides the integration of the infrastructure objects and data sources, by means of appropriate connectors. To this end, a fundamental duty of the MSB is the transformation of the Platform Independent Model (PIM) of the underlying components operational behaviour to the COGLO Platform-Specific Model (PSM). The MSB incorporates also the functionality for the orchestration of COG-LO components and operations as regards the execution of workflows [21]. In addition, the MSB is the main COG-LO system entity for the enforcement of mechanisms for data security, privacy and trust.
The Coordination Layer incorporates the appropriate mechanisms both for the provisioning, management, monitoring and optimization of the Infrastructure Layer as well as for the effective coordination of resources and their actions towards business objectives. A key component of the Coordination Layer is thus the Social Internet of Things.

The Intelligence Layer provides the necessary logical inference mechanisms for knowledge extraction and formalisation, learning and reasoning, as well as cognitive behaviour of the underlying entities. To achieve this, the COG-LO intelligence Layer consists of multiple analytics technologies able to merge and aggregate data from different logistics entities and CLOs, to identify patterns, and to propose operation improvements. Secondly, predictive analytics with event processing enables foreseeing the impact of state changes of one or more operations and determining the corresponding effects on multiple stakeholders. COG-LO Intelligence Layer is coupled with optimization algorithms and heuristics for enabling CLOs adaptation to operational changes from the external environment in near-real time. In particular, COG-LO couples analytics and optimization for considering the effect of optimization control measures to the performance of operations in an environment with continuous external variations.

Finally, the Application Layer consists of the Cognitive Advisor (CA) and the Tweeting CLOs. The CA provides the logistics operator with visual decision support for routing optimisation. The CA interacts with the MSB to visualise the formalisation, reasoning and cognitive outputs of the Intelligence and Coordination Layers. The Tweeting CLO is the generic prototype consisting of the appropriate APIs that receive the messages from the logistics objects and vehicles and transmit them to the CLOs in the same network and to the CA via the MSB. Fostering interoperability, semantic data integration and operational effectiveness, COG-LO components rely on semantic ontologies for grounding data being collected, processed and disseminated, as well as establishing a common understanding between the collaborating entities.

\subsection{Data model}

The COG-LO integrates data from various sources that correspond to stakeholders with different roles in the logistics domain. The provenance of data and the use cases supported by this data exhibit great variety in their nature and cover a broad range of logistics services. Thus, the main challenges faced were (i) the design of a broad homogenized data model, which serves all purposes required, and at the same time (ii) the delimitation of the design to the context of the project.

The context of COG-LO demonstrates a dual nature, namely the physical and the digital one. In the physical context, objects perform normal logistics actions, while in the virtual context they act as virtualized entities with intelligence capabilities. In the physical context, logistics objects are parcels, containers, trucks, ships, trains, 
employees with different duties in the logistics chain, postal offices, warehouses, ports, train stations, etc. The main set of capabilities exhibited by these objects is relevant with handling parcels. Logistics objects are related to organizations for which they execute pick-up and delivery plans. Properties of the logistics objects determine the planning procedure.

In the digital context, the situation is more dynamic. Sensors and smart devices attached to logistics objects, and which are assumed to be networked, transmit information about the underlying objects' state. The information is transmitted via messages that can be sent from one virtual object to the other. The range, to which the messages can be transmitted by a virtual object, is defined by social relationships, which are built and destroyed dynamically, and bear various "friendship" semantics among virtual logistics objects. As receptors of environmental information from the network, virtual objects can assess the state of their environment and cognitively react. Through optimization and decision-making capabilities, they can change their behaviour and suggest analogous actions to their social neighbours. Environmental changes may be populated by other virtual objects or by external components that contribute information to the network via appropriately formed messages encapsulating external events.

The design of the data model of COG-LO followed a topdown approach. Based on the identification of actors and their actions in the logistics setting of COG-LO, the base concepts both in the physical and the digital context were defined. In the physical context, there are four basic data types defined. The parcel is the principal unit of interest of logistics services. The existence of a parcel in the logistics chain starts with an order placed by a customer, and ends when the parcel is delivered to the recipient. Each parcel is attributed by a source location, a destination location, package dimensions and transportation type (e.g. normal, express etc.). The second concept is the container. Containers are placeholders for parcels. They are used to compose parcels into larger cargo units, and they may be encapsulated one inside the other. Besides similar attributes with parcels, containers have also a capacity associated with them, and also they are related with their content, i.e. the parcels or other containers they may carry. Transportation means, is the concept that identifies ships, trains and various types of vehicles. Sea, rail and road are the transportation modes considered in COG-LO, but air transportation could be represented similarly. Similarly to containers and parcels, there is a source and destination assigned to them at each point in time, when they are active in the logistics chain. Capacity is also associated with them and is measured in units corresponding to the amount of containers or parcels they can accommodate. Finally, a station is every establishment where a transportation means may stop and perform a cargo relevant action, that is load, unload, pack, unpack, customs' check etc. Such establishments are warehouses, ports, train stations, postal hubs and offices, end customers, customs and postal boxes.
In the digital context, the concepts of the physical context are mapped to their digital twins. They are attributed with capabilities of four levels: 1. transmit information, 2. receive information, 3 . react to received information and 4. exhibit cognitive behaviour. Based on these capabilities, the digital concepts of VLO and CLO are defined as follows:

\section{VLO definition}

A Virtual Logistics Object is a virtual instance (VI) of a physical logistics object, which is composed by (i) the physical logistics object and (ii) the sensors and smart devices attached to it.

\section{CLO definition}

A Cognitive Logistics Object is a VLO for which the following hold: (i) the corresponding physical logistics object can perform at least one cargo action, (ii) it demonstrates at least 3rd level capabilities.

The Cognitive Logistics Object (CLO) constitutes the bridge between the physical context and the digital context of the COG-LO network. It is a virtual entity that corresponds to exactly one physical resource of the logistics network. For exemplifying the concept, let us assume a truck that transports parcels from one location to another. From the physical perspective, according to our previous analysis of capabilities' levels, it is a passive object. Nevertheless, when the truck is equipped with sensors measuring its weight and capacity, a GPS device tracking continuously its location, and we consider it being driven by a human with a smartphone, the perspective changes. This same object is now classified at least at the 3rd level of capabilities. It can inform constantly the network about the state of its deliveries, it can give an overview of the environment to the driver, and it can also let the driver assess a situation and resolve it, by following recommendations sent, or even by taking decisions based on situational awareness provided.

Each CLO is assigned with a plan. A plan is a sequence of physical actions assigned to the physical counterparts of CLOs. A plan comprises an ordered set of plan steps. Each plan step is executed at a specific location and consists of a series of cargo actions on parcels and containers: (i) loading/unloading, (ii) packing/unpacking (i.e., consolidation of parcels into containers) or (iii) customs checking. A plan step is associated with a cost estimation, which is what makes a plan get selected among a set of alternative ones during the optimization. Each plan is part of a recommendation produced by the Cognitive Advisor. A recommendation is generated as a response to an environmental change of COG-LO.

The COG-LO ontological model was defined, following the standard approach [22]. The development of the COG-LO ontology was performed using the Web Onotology Language (OWL) and the ontology is expressed in RDF/XML syntax. The data model of COG-LO is in detail presented by the COG-LO ontological framework [23]. 


\subsubsection{Data storage, integration and exchanges}

The main sources of information of the COG-LO framework are the databases of the pilot partners. They provide the core data set, based on which all processes of the system are executed. Each data model design, as well as the storage scheme of each source, is different and out of the scope of the project. The information integration is achieved through data connectors that are implemented as part of the Message and Service Bus of COG-LO. They are attached to the data sources and they assume the responsibility of transforming source data into the COG-LO data model.

The core system where the physical data model of COG-LO is deployed is the SIoT infrastructure. The social graph instantiates all virtual instances of the logistics objects, which are retrieved from the pilots' data sources, manipulates them and produces new data, i.e., their social relationships. The properties of the virtual objects as well as their interconnections in the social graph are available in real time to the Cognitive Advisor and the CLOs of the network. Various messages are exchanged (i) among CLOs, (ii) between CLOs and the Cognitive Advisor and (iii) between external event sources (e.g., Traffic Management System) and the Message and Service Bus. The structure of the messages follows the COG-LO data model.

The implementation of the SIoT data storage relies on the Apache Ignite platform [24]. It is a memory-centric distributed database, caching and processing platform for transactional, analytical and streaming workloads delivering in-memory speeds at petabyte scale. The inherent architectural design of Apache Ignite, which employs a distributed approach for both data storage and data caching, made it a natural solution for the implementation of the SIoT platform, where several social Cognitive Logistics Objects are required to be stored and updated in distributed fashion and simultaneously.

The SIoT data infrastructure is organized in a cluster of SIoT peers. One SIoT peer manages the data related to the VIs of the CLOs it is responsible for. Cluster nodes discover each other automatically enabling cluster scaling when necessary. The nodes are divided into two main categories: server and client. Server nodes are storage and computational units of the cluster that hold both data and indexes and process incoming requests along with computations. The platform is based on a durable memory architecture that allows storing and processing data and indexes both in-memory and on-disk, ensuring performance as well as durability.

\subsection{Interoperability}

The vastness of virtualized devices but above all the heterogeneity of their physical counterparts, requires sophisticated techniques to guarantee a high degree of interoperability in terms of communication and interaction.
The Message and Service Bus (MSB) plays a key role in this direction. It acts as the mediation middleware between the various components comprising the COG-LO ecosystem, and is assigned with the interaction, coordination and orchestration of COG-LO components and operations. In that respect, the MSB supports message exchange between system entities, circulation of events, interaction between CLOs. To this end, it facilitates cooperation within communities of CLOs and creation of ad hoc channels between CLOs by enabling the dynamic establishment of message topics.

Another fundamental challenge that the MSB tackles is the transformation of platform independent specifications of the underlying components operational behaviour to the COG-LO platform-specific model. The MSB provides for the integration of infrastructure entities and data sources, by means of data connectors.

In addition, the MSB is the main COG-LO system entity enforcing mechanisms for data security, privacy and trust, and enabling secure orchestration of COG-LO components as regards the execution of the necessary data flows, so that the reference operational scenarios are eventually fulfilled.

The Message and Service Bus provides a set of interfaces for the integration with COG-LO services, components, applications, as well as with external data sources and infrastructure entities:

- Entity management: this interface is used for adding, updating or deleting entities participating in the COG-LO system. It also provides a lookup method for getting details of COG-LO entities.

- Messaging: this interface is used by COG-LO services and applications for data communication. It also allows accessing information provided by various data sources, either internal or external to the COG-LO system.

- Data connector: this interface provides a unified solution for accessing information stored in heterogeneous systems, under a common transactional interface. It enables data interactions with the rest of the platform based on the COG-LO common semantic information model.

Data sources connected to the COG-LO platform (e.g., a traffic management system or public train timetables service) are initially registered to the MSB and data exchanges are handled by the corresponing data connectors. The latter consist of a set of data flows, which publish data to or ingest data from the platform, effectively hiding the implementation details of each data source.

\subsubsection{Data flow management and orchestration}

As part of the Message and Service Bus, the data flow management and orchestration solution enables end users to configure the way COG-LO components interoperate, in order to react to logistics events. It offers a user-friendly 
way to connect COG-LO applications, platforms (e.g., SIoT, smart road infrastructure) and various data sources etc., on the grounds of the abstraction, adaptation and communication features of the MSB. The central point of the solution is its ability to continuously process streams of events and to orchestrate upon event receipt the operations that should be carried out, by which entities and in which order.

The data flow management and orchestration solution of the COG-LO system is based on the Apache NiFi integration platform [25]. Apache NiFi offers a visual command and control centre for designing, testing, deploying and monitoring data flows.

In order to integrate heterogeneous data sources, the following data flows types have been specified:

- Pre-Flow: transforms COG-LO domain requests to data source specific requests.

- Post-Flow: transforms the responses received by the data sources from the data source-specific model to the COG-LO domain model.

- Handler-flow: handles domain requests as they are received from the MSB and communicates with the underlying data source e.g. a database.

- Producer-flow: generates domain events after communicating with the underlying data source.

Outside of the context of a data connector the following data flow types are defined:

- Enforce-flow: processes domain data in a way that access control policies are applied to the data.

- Orchestration-flow: processes domain events and coordinates the chain of operations that need to be performed in order to fulfil given operational needs.

In order for the MSB to respond to an incoming request, for example in a point-to-point communication scenario, the MSB basically builds chains of data flow calls.

The COG-LO data flow management and orchestration solution employs data flows to model the interactions between COG-LO components, enabling end users to create custom data flows for handling incoming events e.g. traffic, emergency, general logistics events. This enables flexible integration of information systems and supports the realization of the COG-LO vision: facilitating the creation of ad hoc logistics collaboration by combining digital processes with physical procedures taking place at the level of actual cargo and means of transportation.

In order to support pilot operations and drive business scenarios a set of orchestrations have been deployed on the COG-LO platform. Their purpose is not limited to order management, but also to provide support for accidental events (e.g. vehicle breakdown) driving dynamic rescheduling of daily deliveries. The orchestrations leverage the Social Internet of Things for this task to intelligently select candidate objects, then contact the Cognitive Advisor to receive updated plans for the vehicles involved and finally initiate the negotiation orchestration.

\subsection{Security, privacy and trust}

The MSB as the core communication module of the system, enables access to both internal and external services and information through a unified interface. In this context, the mechanisms for security, privacy and trust cover all respective technologies, notably access and usage control, cryptography and trust infrastructure. Specifically, COG-LO provides a solution for identity management offering standard-based means for authenticating COG-LO actors; a policy-based access and usage control framework for regulating the circulation and usage of information. It additionally provides an architecture with a standard set of components, such as Policy Administration Point (PAP), Policy Decision Point (PDP), Policy Information Point (PIP) and Policy Enforcement Point (PEP) for evaluating access control policies.

The COG-LO platform adopts a token-based authentication solution offered by RedHat's KeyCloak identity and access management component [26], and is used for both user authentication as well as component (service) authentication.

For what concerns the access control, COG-LO adopts the Attribute-based Access Control (ABAC) paradigm and is established upon the XACML 3.0 language and reference architecture. In particular, the starting point for incorporating $\mathrm{ABAC}$ authorisation functionality within the MSB has been extensible Access Control Markup Language (XACML) that has been extensively used in academia and industry.

In line with the XACML reference architecture, the MSB, as the PEP, provides the mechanisms for enforcing the specified access and usage control policies when it comes to regulating message exchange between COG-LO components, services or CLOs. The MSB interacts with the PDP by providing attributes obtained by the original request, with the latter transformed in the XACML format that the PDP can process.

In the context of COG-LO, AuthZForce [29] has been selected as the policy decision engine as it implements the OASIS XACML 3.0 core specification, and provides an API to get authorisation decisions, based on authorisation policies, and authorisation requests from PEPs.

Cryptography traditionally represents the bottom line of data protection. Therefore, COG-LO puts in place a rich functional toolkit able to support all necessary cryptographic functionalities to foster data confidentiality. To this end, the COG-LO crypto-engine leverages a plethora of cryptographic primitives, both symmetric and asymmetric. Furthermore, COG-LO adopts the advanced technology of Attribute-Based Encryption (ABE), targeting the cryptographic enforcement of data disclosure policies by leveraging the attributes assigned to entities, being people or systems. 
Concerning aspects such as secure channel establishment, COG-LO identified a series of shortcomings to the cornerstone technologies that facilitate secure information exchange over the Internet, namely the Public Key Infrastructure (PKI) and associated X.509 certificate standard [30]. Specifically, as it has been recently shown, PKIs are exposed to risks due to errors or breaches involving Certification Authorities (CAs), resulting in unauthorised certificates being issued and compromising thus the security of the corresponding end users. In light of the above, COG-LO adopts a novel blockchain-based solution enabled by the Hyperledger [27] family of technologies, namely the Hyperledger Indy and the Hyperledger Aries frameworks in order to establish secure crossorganisational communication. The aforementioned solution, being based on the blockchain technology, inherits inevitably its advantages. The solid basis of the public append-only log (past logs cannot be changed unless the blockchain is subverted by a dishonest network majority), eliminate the single-point-of-failure issue and enables rapid reaction to identity revocations since DIDs can be validated on the distributed ledger.

\section{PERFORMANCE ANALYSIS}

The fundamental aspect that is used to evaluate the performance of the COG-LO framework is the time consumption of the algorithms implemented within the Social Internet of Things (SIoT) and the optimizer.

More specifically, the scalability of these components is addressed by observing how the computational time for object digitalization varies as the number of objects increases, how the social graph surfing time varies when a friend must be discovered as the size of social graph varies, and finally by the complexity of the optimization algorithms in large environments, where the problem of a large amount of data and variables to be analyzed must be faced.

The Social Internet of Things (SIoT) architecture consists of various SIoT clusters. Each cluster is implemented using Apache Ignite to support a memory-centric distributed database, caching and processing platform [17]. Each SIoT peer can automatically discover each other in order to create a cluster or to browse another peer's social graph. In the first performance study of the SIoT, the response time for the creation of a SIoT social graph was observed, in relation to the size of the graph (number of CLOs). The initialization process entails the instantiation of the digital twins of logistics objects, along with their relevant data structures [28]. Fig. 3 demonstrates how this process scales in time increasing the number of CLOs forming the social graph.

The SIoT graph receives update requests every time the state of CLOs changes. These updates require recalculating the relationships between the CLOs. In this experiment, the time required for a CLO state update and the creation of social relationships between a CLO and $\mathrm{N}$ friends CLOs is observed. Fig. 4 depicts the results and demonstrates the time necessary to modify the data structures of both the CLO that wants to establish a friendship and the data structures of all involved friends (since the social relations are bidirectional).

As usual in traditional distributed deployments of systems like the SIoT platform used in this project, different servers are used to share the load of traffic and computation. Each server can be configured to work following a full replication or a partial replication scheme, or even as a totally independent system with non-replicated data. Using the first approach all the data is replicated or copied to all the participating nodes in the cluster. Otherwise, using the second approach, the entire data is split equally into partitions and is stored in the participating nodes, thereby creating a distributed storage of data. The total storage space depends on the total memory available across the peer. As shown in Fig. 5, the replicated mode allows the speed-up of the discovery process, since the information is immediately available in the peer from which the search is being performed. While this approach has benefits in terms of time, it also requires an increased use of resources.

The optimization performed by the CA is based on the graph representation of the CLOs network. Upon the occurrence of a disruption event (e.g., an ad hoc order, a traffic event etc.), the graph gets pruned by SIoT to include only CLOs in the vicinity of the event. The size of the graph, i.e., the number of vehicles included in the optimization impacts greatly the response time.

The performance of the optimization algorithms is presented in Table 1. It clearly shows the importance of graph pruning to achieve real-time responsiveness to disruption events. Table 1 shows the performance time for optimization processing on pruned graphs. An optimization algorithm uses exact methods, with linear solver, where using a large number of CLOs exponentially increases processing time. For optimization processing, the total infrastructure graph is clustered into regional representation with graph sizes of 300-500 CLOs (postal offices, vehicles, parcels). The processing time in Table 1 clearly shows that without pruning the graph and omitting the number of CLOs included in event handling, the system would not be able to create real-time responses.

Table 1 - VRP optimization response time, based on the number of CLOs included

\begin{tabular}{|c|c|c|c|c|}
\hline vehicles/post offices & $\mathbf{2 0}$ CLOs & $\mathbf{2 5}$ CLOs & $\mathbf{3 0}$ CLOs & $\mathbf{4 0}$ CLOs \\
\hline $\mathbf{2}$ CLOs & $4.9 \mathrm{~s}$ & $12.4 \mathrm{~s}$ & $28.7 \mathrm{~s}$ & $95.1 \mathrm{~s}$ \\
\hline $\mathbf{3}$ CLOs & $9.9 \mathrm{~s}$ & $26.3 \mathrm{~s}$ & $43.4 \mathrm{~s}$ & $168.3 \mathrm{~s}$ \\
\hline $\mathbf{4}$ CLOs & $18.1 \mathrm{~s}$ & $38.2 \mathrm{~s}$ & $78.5 \mathrm{~s}$ & $258.2 \mathrm{~s}$ \\
\hline $\mathbf{5}$ CLOs & $27.5 \mathrm{~s}$ & $52.6 \mathrm{~s}$ & $127.2 \mathrm{~s}$ & $378.4 \mathrm{~s}$ \\
\hline $\mathbf{6}$ CLOs & $40.7 \mathrm{~s}$ & $117.4 \mathrm{~s}$ & $228.4 \mathrm{~s}$ & $592.2 \mathrm{~s}$ \\
\hline $\mathbf{7 ~ C L O s}$ & $52.8 \mathrm{~s}$ & $172.1 \mathrm{~s}$ & $415.6 \mathrm{~s}$ & $865.4 \mathrm{~s}$ \\
\hline $\mathbf{8}$ CLOs & $74.3 \mathrm{~s}$ & $230.6 \mathrm{~s}$ & $720.1 \mathrm{~s}$ & $1923.3 \mathrm{~s}$ \\
\hline
\end{tabular}




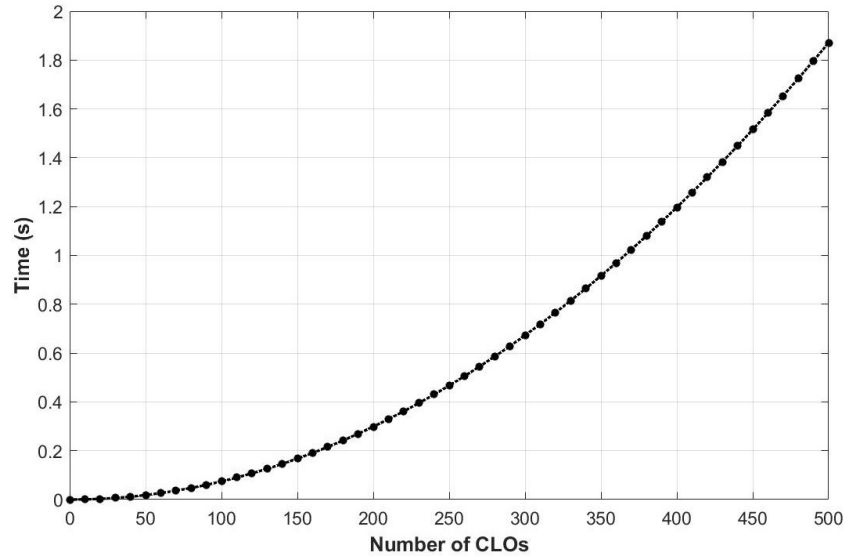

Fig. 3 - Required time for digital twin creation and SIoT graph initialization varying the number of CLOs

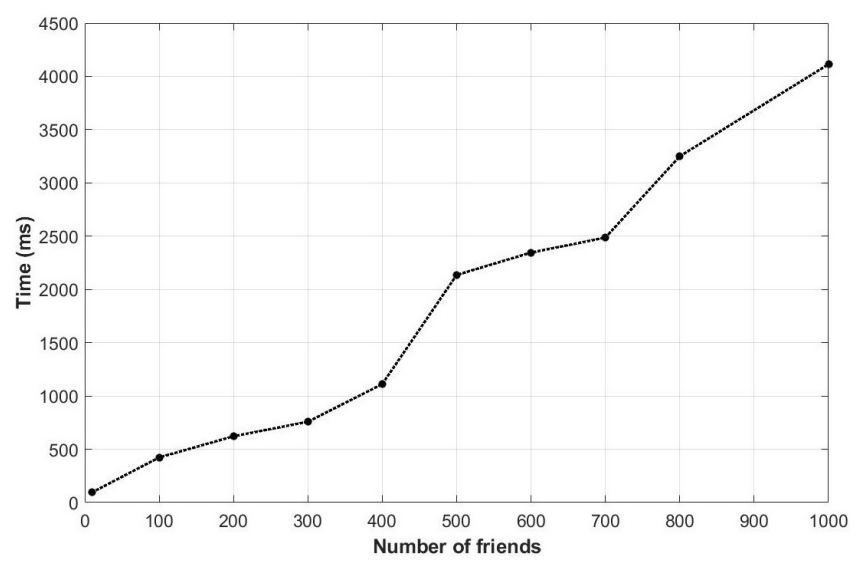

Fig. 4 - Time consumption for SIoT graph updates on creating new friendships between a CLO and varying number of friends

\section{CONCLUSION}

In this article, the innovative concept of the Cognitive Logistics Object, introduced within the COG-LO project, is presented. CLOs represent all the entities involved in logistics processes. They are made autonomous and dynamically reactive to the surrounding environment thanks to their cognitive capabilities. CLOs are able to collaborate and implement functionalities stemming from social networks thanks to virtualization techniques and the properties of the Social Internet of Things (SIoT) framework that has been exploited. The efficient architecture that implements the COG-LO framework has experimentally been tested, demonstrating that the proposed solution allows for optimization operations in responsive time outperforming in-place logistics procedures.

The results achieved through the application of SIoT and the relevant virtualization techniques are encouraging. The significant benefits in terms of interoperability, motivates for exploring the topic on a large scale. The adoption of the SIoT and the CLO concept proves to effectively mit- igate the optimization problem complexity, which allows the use of classical optimization techniques for real-time events processing. The exploitation of SIoT and CLO used in the COG-LO project offers an effective approach in managing new emerging problems in logistics infrastructure.

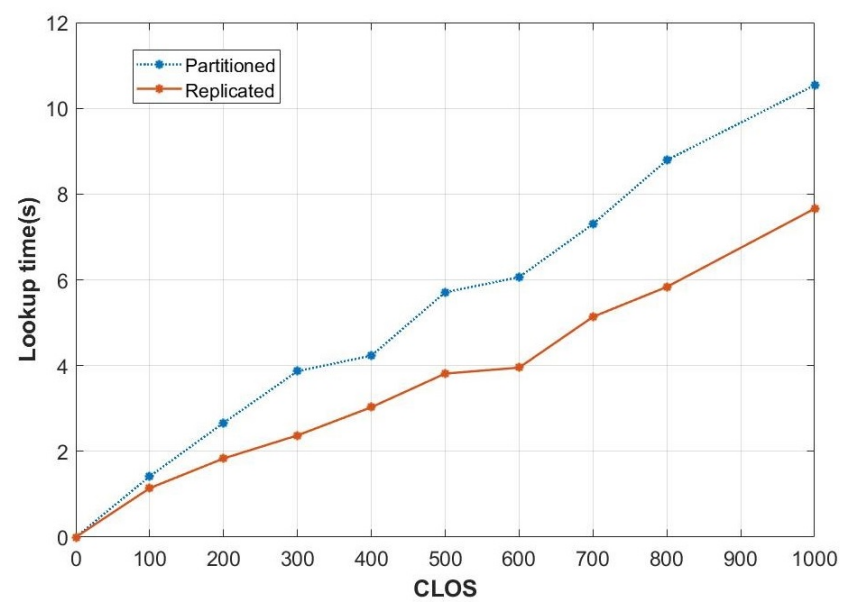

Fig. 5 - SIoT lookup time varying the number of CLO friends searched for replicated vs partitioned memory mode

\section{ACKNOWLEDGEMENT}

This work was partially supported by the European Union's Horizon 2020 research and innovation program under the COG-LO project (grant agreement no. 769141).

\section{REFERENCES}

[1] Walid, Taha, Abd-Elhamid, Thunberg, Johan, What is a Cyber-Physical System?, 10.1007/978-3-030-360719-1, 2020.

[2] http://www.cog-lo.eu/.

[3] Evtodieva, T. Chernova, D. Ivanova, N. Kisteneva, Logistics 4.0, 10.1007/978-3-030-11754-2-16, 2019.

[4] L. Atzori, et al., The social internet of things (SIoT) when social networks meet the internet of things: Concept, architecture and network characterization, Computer Networks 56(16), pp. 3594-3608, 2012.

[5] S. Belardo, H. L. Pazer, Scope/Complexity: A Framework for the Classification and Analysis of InformationDecision Systems, J. of Management Information Systems 2(2): 55-72 (2015).

[6] Psaraftis HN. Dynamic vehicle routing: Status and prospects, Annals of Operations Research, 61:143164, 1995.

[7] GS1, Logistics Interoperability Model, Version 1, Issue 1.0, http://www.gs1.org/lim, August 2007.

[8] Pillac, Victor, et al., A review of dynamic vehicle routing problems, European Journal of Operational Research 225.1: 1-11, 2013.

[9] M. Grieves, Digital twin: Manufacturing excellence through virtual factory replication, 2014. 
[10] M. Heutger and M. Kuechelhaus, Digital twins in Logistics: a DHL perspective on the impact of digital twins in the logistics industry, DHL, 2019.

[11] R. Minerva, G. M. Lee and N. Crespi, Digital Twin in the IoT Context: A Survey on Technical Features, Scenarios and Architectural Models, Proceedings of the IEEE, vol. 108, no. 10, October 2020.

[12] Gartner, Gartner Identifies the Top 10 Strategic Technology Trends for 2019, 2018: https://www.gartner.com/en/newsroom/pressreleases/2018-10-15-gartner-identifies-the-top-10strategic-technology-trends-for-2019.

[13] K. Kalaboukas, G. Lioudakis, M. Koukovini, E. Papagiannakopoulou, G. Morabito , N. Dellas, M. Zacharias, S. Quattropani, M. Samarotto, M. Jermol, M. Cimperman, L. Stopar, M. Senozetnik, S. Bratusa, A. Kahvedzic, D. Justament, I.-K. Buntic, H. Marentakis, A. Maragakis, E. Al, E. Özsalih, A. Kiousi, A. Dimitriou, G. Galli, E. Pastori, E. Gualandi, F. Alesiani, G. Ermis, T. Jacobs, I. Mourtos, S. Lounis and G. Zois, Cognitive Logistics Operations through Secure, Dynamic and adhoc Collaborative Networks: The COG-LO project, London, 2019.

[14] M. Nitti, et al., The virtual object as a major element of the internet of things: a survey, IEEE CS and T, 18(2), pp. 1228-1240, 2016.

[15] L.Atzori, C. Campolo, et. al., Enhancing Identifier/Locator Splitting through Social Internet of Things, IEEE Internet of Things Journal, 2018.

[16] J. Travers, S. Milgram, An experimental study of the small world problem. Sociometry, 32, pp. 425-443, 1969.

[17] http://www.cog-lo.eu/sites/default/files/documents/ 2020-05/COG-LO-D3.6-COG-LO architecture definition (final version) - v1.0.pdf

[18] Atzori Luigi, Campolo Claudia, Iera Antonio, Milotta G., Morabito Giacomo, Quattropani, S., Sociocast: Design, Implementation and Experimentation of a New Communication Method for the Internet of Things, 662-667. 10.1109/WF-IoT.2019.8767348, 2019.

[19] He, Ruhan, et al., Balanced k-means algorithm for partitioning areas in large-scale vehicle routing problem, 2009 Third International Symposium on Intelligent Information Technology Application. Vol. 3. IEEE, 2009.

[20] Bent, Russell, and Pascal Van Hentenryck, Spatial, temporal, and hybrid decompositions for large-scale vehicle routing with time windows. International Conference on Principles and Practice of Constraint Programming. Springer, Berlin, Heidelberg, 2010.
[21] http://www.cog-lo.eu/sites/default/files/documents/ 2020-11/D4.4 Coordination Mechanisms (final).pdf

[22] S. Negru, F. Haag, and T. Ertl S. Lohmann, Visualizing Ontologies with VOWL, Semantic Web Journal, 2015.

[23] http://www.cog-lo.eu/sites/default/files/documents/ 2020-05/COG-LO-D3.4-COG-

LO_dataModel_ontologicalFramework-final.pdf

[24] https://ignite.apache.org/

[25] https://nifi.apache.org/

[26] https://www.keycloak.org/

[27] https://www.hyperledger.org/

[28] http://www.cog-lo.eu/sites/default/files/documents/ 2020-02/COG-LO-D3.3-COG-LO_dataModel_ ontologicalFramework-final.pdf

[29] https://authzforce.ow2.org/

[30] Internet X.509 Public Key Infrastructure Certificate and Certificate Revocation List (CRL) Profile, IETF RFC 5280, May 2008, https://tools.ietf.org/html/rfc5280.

\section{AUTHORS}

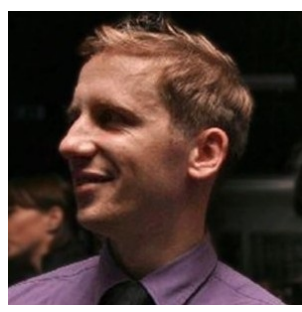

Miha Cimperman, Ph.D, is a researcher and project lead at the Jozef Stefan Institute, Laboratory for Artificial Intelligence, Slovenia. His work focuses on research in the field of knowledge services development for critical infrastructure, namely: logistics, energy and finance infrastructure. His research is focused on data modelling and analytical solutions design based on sensor IoT data and text data.

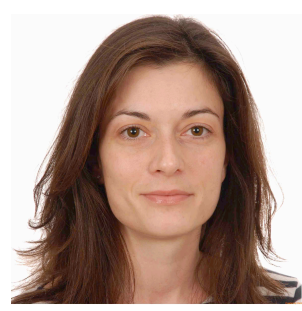

Angela Dimitriou, is an Electrical and Computer Engineer, graduated from the National Technical University of Athens, Greece. She received her Ph.D. in the scientific domain of databases, collaborating with the Knowledge and Database Systems Laboratory of NTUA, Greece. Her research interests include data management, semi-structured data, keyword search, information retrieval, graph data and algorithms. She works at the National Technical University of Athens and has been collaborating with INTRASOFT International for several European research projects. 


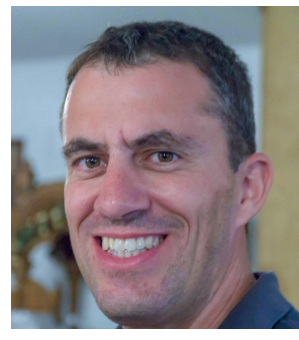

Kostas Kalaboukas, is Technology Transfer and New Business Development Manager at Gruppo Maggioli and candidate Ph.D student at the Technical University of Crete. His research focuses on digital supply chains and smart cities and has participated in many research projects in the past. He has studied Production Engineering and Management at the Technical University of Crete (1991-1996) and obtained an MSc in Total Quality Management at the University of Piraeus (2000). He has wide experience in business analysis, quality assurance and management of complex software projects.

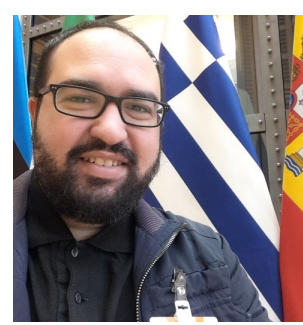

Aziz S. Mousas, received his diploma in Applied Mathematics from the school of Applied Mathematics and Physics of the National Technical University of Athens (NTUA) in 2008, and his Dr.-Ing. degree in Electrical and Computer Engineering from NTUA in 2014. He has participated in several European and national R\&D projects as a researcher, software engineer, project manager and ICT consultant.
His research and professional interests include security and privacy protection, software engineering, middleware and distributed systems, as well as the applications of semantic ontologies in these fields. He has more than 20 publications in international journals, conferences and books related to these areas, as well as contributions to the MPEG-21 and MPEG-M ISO standards.

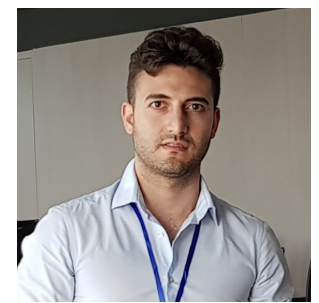

Salvatore Quattropani, received BSc. and MSc. degrees in Computer Engineering in 2016 and 2017, respectively, from University of Catania, Italy. Since August 2017 he is with the Consorzio Nazionale Interuniversitario per le Telecomunicazioni as a research engineer. His research interests focus on IoT connectivity and computing approaches. 